\title{
A New Glimm Functional and Convergence Rate of Glimm Scheme for General Systems of Hyperbolic Conservation Laws
}

\author{
Jiale Hua $^{1}$ Zaihong Jiang ${ }^{2}$ and Tong Yang ${ }^{1}$ \\ ${ }^{1}$ Department of Mathematics \\ City University of Hong Kong \\ Kowloon,Hong Kong \\ ${ }^{2}$ Joint Advanced Research Center in Suzhou \\ University of Science and Technology of China \\ and City University of Hong Kong \\ Suzhou,Jiangsu
}

\begin{abstract}
In this paper, we introduce a new Glimm functional for general systems of hyperbolic conservation laws. This new functional is consistent with the classical Glimm functional for the case when each characteristic field is either genuinely nonlinear or linearly degenerate so that it can be viewed as "optimal" in some sense. With this new functional, the consistency of the Glimm scheme is proved clearly for general systems. Moreover, the convergence rate of the Glimm scheme is shown to be the same as the one obtained in [6] for systems with each characteristic field being genuinely nonlinear or linearly degenerate.
\end{abstract}

\section{Introduction}

There have been extensive studies on the mathematical theory for the systems of hyperbolic conservation laws. One of the typical examples of these systems is the compressible Euler equations for fluid dynamics. As for the Cauchy problem, 
the celebrated paper [13] by Glimm in 1965 established the global existence of weak solutions with small total variation under the assumption that each characteristic field is either genuinely nonlinear or linearly degenerate. Even though the system of compressible Euler equations for gas dynamics satisfies this assumption, there are many other physical systems such as those arising from elasticity and magneto-hydrodynamics whose characteristic fields do not all satisfy this assumption. To generalize the Glimm theory to the general systems, the key component is the re-definition of the Glimm functional for wave interactions in the same characteristic family. For this, a cubic functional was introduced by Liu in [22] and was later elaborated by Liu-Yang in [24] through the introduction of an effective angle between two waves in the same family. This improvement is successful in establishing the existence, but it is less satisfactory for the consistency and the convergence rate analysis. Therefore, the main purpose of this paper is to introduce a new Glimm functional for wave interactions in the same family for the general system so that the Glimm theory can now be presented in an elegant way.

Consider the Cauchy problem for a system of hyperbolic conservation laws

$$
\begin{cases}u_{t}+f(u)_{x}=0, & t \geq 0,-\infty<x<\infty, \\ u(x, 0)=u_{0}(x), & -\infty<x<\infty\end{cases}
$$

where $u \in \mathbb{R}^{n}, f: \Omega \mapsto \mathbb{R}^{n}$ is a smooth vector field with $\Omega \subset \mathbb{R}^{n}$ being an open set. Denote $A(u)=D f(u)$ the $n \times n$ Jacobian matrix of the flux function $f$. One of the main features of this type of systems is that discontinuities will form in finite time no matter how smooth the initial data is except for some special cases. This leads to the development of the shock wave study in which many fundamental theories have been established, cf. [1, 4, 7, 11, 12, 13, 14, 19, 17, $26,27]$ and references therein. In the framework of solutions with small total variation, the global existence of solutions was proved in the fundamental work of Glimm by introducing the Glimm scheme and by using the solutions to the Riemann problems solved by Lax as building blocks, while the stability in $\mathbf{L}^{1}$ norm was obtained much later, cf. $[1,5,7,20,24]$ and the references therein. As related, we mention the recent breakthrough made by Bianchini-Bressan on the vanishing viscosity for solutions in this class for hyperbolic systems with artificial viscosity.

For the later presentation, we now introduce some notations. As usual, the 
system (1.1) is called strictly hyperbolic, if for every $u \in \Omega$, the matrix $A(u)$ has $\mathrm{n}$ real distinct eigenvalues denoted by

$$
\lambda_{1}(u)<\lambda_{2}(u)<\ldots<\lambda_{n}(u) .
$$

Corresponding to these eigenvalues, there are $n$ linearly independent right eigenvectors

$$
r_{1}(u), r_{2}(u), \ldots, r_{n}(u) .
$$

To capture the nonlinearity of these characteristic fields, the following definition is from [19].

Definition 1.1. For each $i \in\{1,2, \ldots, n\}$, the $i$-th characteristic field is called genuinely nonlinear, if

$$
\nabla \lambda_{i} \cdot r_{i} \neq 0, \text { for all } u \in \Omega \text {. }
$$

While the $i$-th characteristic field is called linearly degenerate, if

$$
\nabla \lambda_{i} \cdot r_{i} \equiv 0, \text { for all } u \in \Omega
$$

As mentioned earlier, in general, the solution to the Cauchy problem (1.1) develops singularity in the form of discontinuity, called shock, because of the nonlinearity of the flux function $f(u)$. Thus, solution to (1.1) is defined in the weak sense as follows.

Definition 1.2. A function $u:[0, T] \times \mathbb{R} \longmapsto \mathbb{R}^{n}$ is a weak solution of the problem (1.1), if $u$ is a bounded measurable function and

$$
\iint_{t \geq 0}\left[u \phi_{t}+f(u) \phi_{x}\right] \mathrm{d} x \mathrm{~d} t+\int_{t=0} u_{0}(x) \phi(x, 0) \mathrm{d} x=0,
$$

holds for any smooth function $\phi$ with compact support in $\left\{(x, t) \mid(x, t) \in \mathbb{R}^{2}\right\}$.

To solve the Cauchy problem (1.1), Glimm introduces a scheme to construct the solution to systems with the following assumption [13]:

Each characteristic field is either genuinely nonlinear or linearly degenerate.

There are two main ingredients in the Glimm scheme. One is the Glimm functional which we will discuss in the next section. The other one is to approximate the initial data by piecewise constant functions and solve the Riemann 
problems locally in space and time. Here, the Riemann problem is the problem (1.1) when the initial data is given by:

$$
u_{0}(x)=\left\{\begin{array}{lll}
u^{-} & \text {if } & x<0 \\
u^{+} & \text {if } & x>0
\end{array}\right.
$$

where $u^{ \pm}$are constants.

The Glimm functional is used to guarantee that the total variation of the solution is of the same order of the one for the initial data so that the solutions to the Riemann problems solved locally in space and time can be used as building blocks for the construction of the weak solution. In addition, the uniform boundedness in the total variation of the approximate solutions in the Glimm scheme leads to the convergence of the approximate solutions to the global entropy solution as the grid size tends to zero.

In the Glimm scheme, the convergence is in the sense of almost everywhere with respect to the measure in the space of the random sequences. To make this kind of convergence deterministic, a wave tracing argument was introduced in [21] and it was shown that the approximate solution converges as long as the random sequence is chosen to be equidistributed. In the deterministic version of the Glimm scheme with the wave tracing argument, physical waves are divided into virtual waves in the scheme which can be either traced back, or cancelled or created in a short time interval. The wave pattern is greatly simplified if we keep only those waves that can be traced back and replace them by the ones with the same strengths and fixed propagation speeds in some small time interval. If the random sequence is equidistributed, the error due to this simplification can be controlled by the Glimm functional times a small factor related to the grid size, and this factor converges to zero in $\mathbf{L}^{1}$ norm when the grid size tends to zero. Here the equidistributed sequence is defined as follows.

Definition 1.3. A sequence $\left\{\theta_{i}\right\}_{i=0}^{\infty}$ in $[0,1]$ is called equidistributed if

$$
A(N, I) \equiv\left|\frac{B(N, I)}{N}-\right| I|| \rightarrow 0, \quad \text { as } N \rightarrow \infty,
$$

for any subinterval I of $[0,1]$. Here $B(N, I)$ denotes the number of $i, 1 \leq i \leq N$, such that $\theta_{i} \in I$ and $|I|$ is the length of $I$.

In fact, the complement of the set of equidistributed sequences has measure zero. And the equidistributed sequence leads to a clear description of the 
structure of the weak solution through the wave tracing argument. As one step further, to study the convergence rate of the Glimm scheme as the grid size tends to zero for general entropy solutions, the following sequence is used, cf. $[6]$.

Lemma 1.1. Let

$$
D_{m, n}=\sup _{\lambda \in[0,1]}\left|\lambda-\frac{1}{n-m} \sum_{m \leq l<n} \chi_{[0, \lambda]}\left(\theta_{l}\right)\right|,
$$

then there exists a sequence $\left\{\theta_{l}\right\}_{l \geq 0} \subset[0,1]$ such that

$$
D_{m, n} \leq O(1) \frac{1+\ln (n-m)}{n-m} \quad \forall n>m \geq 1 .
$$

By applying the new Glimm functional to the study of the convergence rate, we need to use the $L^{1}$ stability of the standard Riemann semigroup generated by (1.1), denoted by $\left\{S_{t} ; t \geq 0\right\}$. The breakthrough on the $L^{1}$ stability of the weak solutions to (1.1) was first made in [3] for $2 \times 2$ systems and was settled satisfactorily in $[5,7,24]$ for systems satisfying the condition (A). The $L^{1}$ stability of entropy solutions for general hyperbolic conservation laws was later proved in [1] through the vanishing viscosity argument. In fact, [1] considers the Cauchy problem for the hyperbolic system with artificial viscosity

$$
u_{t}+A(u) u_{x}=\epsilon u_{x x}, \quad u(0, x)=u_{0}(x) .
$$

Assume that the matrix $A(u)$ is strictly hyperbolic, smoothly depending on $u$ in a neighborhood of a compact set $K \subset \Omega \subset \mathbb{R}^{n}$. Then there exist constants $c$, $L, L^{\prime}$ and $\delta>0$ such that the following holds. If

$$
\text { T.V. } u_{0}<\delta, \quad \lim _{x \rightarrow-\infty} u_{0}(x) \in K,
$$

where T.V. means the total variation in $x$ variable, then for each $\epsilon>0$ the Cauchy problem (1.8) has a unique solution $u^{\epsilon}$, defined for all $t \geq 0$, denoted by $u^{\epsilon}=u^{\epsilon}(t, x)=S_{t}^{\epsilon}\left(u_{0}\right)$. In addition,

$$
\begin{aligned}
& \text { T.V.S } S_{t}^{\epsilon} u_{0} \leq C T . V . u_{0}, \\
& \left\|S_{t}^{\epsilon} u_{0}-S_{t}^{\epsilon} v_{0}\right\|_{L^{1}} \leq L\left\|u_{0}-v_{0}\right\|_{L^{1}}, \\
& \left\|S_{t}^{\epsilon} u_{0}-S_{s}^{\epsilon} u_{0}\right\|_{L^{1}} \leq L^{\prime}(|t-s|+\mid \sqrt{\epsilon t}-\sqrt{\epsilon s \mid}) .
\end{aligned}
$$


Moreover, when $\epsilon \rightarrow 0+$, the solution $u^{\epsilon}$ converge to the trajectory of a semigroup $S_{t}$ such that

$$
\left\|S_{t} u_{0}-S_{s} v_{0}\right\|_{L^{1}} \leq L\left\|u_{0}-v_{0}\right\|_{L^{1}}+L^{\prime}|t-s|
$$

These vanishing viscosity limits can be regarded as the unique vanishing viscosity solutions of the hyperbolic Cauchy problem

$$
u_{t}+A(u) u_{x}=0, \quad u(0, x)=u_{0}(x) .
$$

In the conservative case when $A(u)=D f(u)$, every vanishing viscosity solution is a weak solution of (1.1) satisfying the entropy condition.

Furthermore, under the condition (A), the vanishing viscosity solutions coincide with the unique limits of the Glimm and front-tracking approximations. In this paper, we will not touch the $L^{1}$ stability or the uniqueness of the weak solutions to the general hyperbolic conservation laws through the Glimm scheme. Instead, we assume that for any two nearby initial data, the unique semigroup generated by the Glimm scheme $S_{t}$ satisfies

$$
\left\|S_{t} \bar{u}-S_{t} \bar{v}\right\|_{\mathbf{L}^{1}} \leq L\|\bar{u}-\bar{v}\|_{\mathbf{L}^{1}}, \quad \forall \bar{u}, \bar{v} \in \mathcal{D}, t \geq 0
$$

for some uniform constant $L$.

Based on the $L^{1}$ stability (1.10), under the condition (A), the convergence rate is shown to be o(1) $\sqrt{s}|\ln (s)|$, cf. [6]. Here $s$ is the grid size in the Glimm scheme. And this convergence rate will be shown to be the same for general hyperbolic conservation laws by using the new functional introduced in this paper.

For general systems, the solution to Riemann problem has different structure so that the Cauchy problem has richer nonlinear phenomena. To capture the wave interactions, the Glimm functional for waves in different families is the same while the one for waves in the same family is different. For this purpose, one cubic functional was introduced in [22] which was elaborated in [25] to take care of the wave interactions globally. The functional used in [25] is defined by the product of the strengths of two involved waves and their effective "interaction" angle. Based on this improvement, the complete existence theory with the wave tracing argument for general systems was obtained in [25] under the assumption: 
For each characteristic field, the linear degeneracy manifold $L D_{i} \equiv\left\{u: \nabla \lambda_{i}(u) \cdot r_{i}(u)=0\right\}$ either is the whole space or $(B)$ consists of a finite number of smooth manifolds of codimension one, which are transversal to the characteristic vector $r_{i}(u)$.

Even though the improved Glimm functional used in [25] is effective in the study of the existence of entropy solutions, it is not satisfactory in proving the consistency and the convergence rate of the Glimm scheme. In fact, the consistency of the Glimm scheme was proved in [25] by carefully and artificially dividing the waves into groups according to their wave strengths compared with the grid size to some power, and the convergence rate of the Glimm scheme was shown to be $o(1) s^{\frac{1}{4}}|\ln s|$ in [29], and then $o(1) s^{\frac{1}{3}}|\ln s|$ in [15] which are less compared to the one given in [6] under the condition (A).

The new Glimm functional for the wave interactions in the same family is optimal in the following sense. First, it yields a clear and complete proof of the consistency of the Glimm scheme. Then it leads to the proof of the same order convergence rate for the general systems as the one under the condition (A). Finally, it will be shown to have the same decrease effect as the classical one introduced by Glimm when the assumption of genuine nonlinearity is imposed. Therefore, the Glimm scheme for the general systems can be analyzed satisfactorily without any artificial adjustment.

The convergence rate of the Glimm scheme can be stated as follows.

Theorem 1.1. Let $\left\{\theta_{m}\right\}_{m=1}^{\infty}$ be a sequence of numbers in $[0,1]$ satisfying (1.7). Given any initial condition $\bar{u}$ with small total variation, let $u(\cdot, t)=S_{t} \bar{u}$ be the unique solution of (1.1), and let $u^{s}$ be the corresponding Glimm approximation solution with grid size $s$ in time direction, generated by the sampling sequence $\left\{\theta_{m}\right\}_{m=1}^{\infty}$. Then for every $T \geq 0$,

$$
\lim _{s \rightarrow 0} \frac{\left\|u^{s}(\cdot, T)-u(\cdot, T)\right\|_{L^{1}}}{s^{\frac{1}{2}}|\ln s|}=0 .
$$

The limit is uniform with respect to $\bar{u}$, as long as T.V.ū remains uniformly small.

Finally in the introduction, we mention the corresponding results on the convergence rates for the vanishing viscosity. Let the system (1.1) be strictly hyperbolic and the condition (A) hold. Then, given any initial data $u(0, x)=$ $u_{0}(x)$ with small total variation, for every $\tau>0$ the corresponding solutions 
$u, u^{\varepsilon}$ of (1.1) and (1.8) were shown to satisfy the following estimate in [9]

$$
\left\|u^{\varepsilon}(\tau, \cdot)-u(\tau, \cdot)\right\|_{L^{1}}=O(1) \cdot(1+\tau) \sqrt{\varepsilon}|\ln \varepsilon| T \cdot V \cdot u_{0}(x) .
$$

This convergence rate is "optimal" in the sense that even for a scalar conservation law, the method of Kuznetsov in [18] shows that the convergence rate is $O(1) \cdot \varepsilon^{1 / 2}$ which is sharp, cf. [28]. The factor $|\ln \varepsilon|$ comes from the interaction of waves in different families in the system which does not exist for scalar equation.

The rest of the paper will be organized as follows. In the next section, the new functional is introduced together with some preliminaries of the wave interaction estimates in the wave tracing argument. The non-increasing property of the new Glimm functional and its application to the consistency of the Glimm

scheme will be proved in Section 3. And the convergence rate of the Glimm scheme stated in Theorem 1.1 will be proved in the last section.

\section{Glimm Scheme and New Functional}

The Riemann problem under the general condition (B) is much more complicated than the case under the condition (A). The Lax entropy condition used under the condition (A) should be replaced by the following Liu's entropy condition under the condition (B).

Definition 2.1. [23] A discontinuity $\left(u_{-}, u_{+}\right)$is admissible if

$$
\sigma\left(u_{-}, u_{+}\right) \leq \sigma\left(u_{-}, u\right)
$$

for any state $u$ on the Hugoniot curve $H\left(u_{-}\right)$between $u_{-}$and $u_{+}$, where $H\left(u_{-}\right) \equiv$ $\left\{u: \sigma\left(u_{-}-u\right)=f\left(u_{-}\right)-f(u)\right\}$.

Corresponding to the $\mathrm{n}$ characteristic fields of the system, there are $\mathrm{n}$ Hugoniot curves. Any state $u$ on the i-th Hugoniot curve $H_{i}\left(u_{0}\right)$ is connected to $u_{0}$ by an i-th shock wave, if the above entropy condition is satisfied. We denote $H_{i}(\alpha)\left(u_{0}\right)$ the state which can be connected to $u_{0}$ by an i-th shock wave with strength $\alpha$. Note that the shock wave described here include the case of contact discontinuity.

Another basic wave pattern used to solve Riemann problem is called the rarefaction wave. For each characteristic field, the state $R_{i}(\alpha)\left(u_{0}\right)(i=1,2 . \cdots, n)$ 
is connected to $u_{0}$ by an i-th rarefaction wave with strength $\alpha$, if

$$
\left\{\begin{array}{l}
\frac{d}{d \alpha} R_{i}(\alpha)\left(u_{0}\right)=r_{i}\left(R_{i}(\alpha)\left(u_{0}\right)\right), \quad \text { for } \lambda_{i}\left(R_{i}(\alpha)\left(u_{0}\right)\right)>\lambda_{i}\left(u_{0}\right) \\
R_{i}(0)\left(u_{0}\right)=u_{0}
\end{array}\right.
$$

Here the wave strength $\alpha$ is used as a parameter along the rarefaction wave curve.

By implicit function theorem, the Riemann problem for general systems is solved by piecing together waves in different families. And with the Liu's entropy condition, each wave in the i-th family, called i-wave may be the composition of several i-th admissible shocks and rarefaction waves.

Suppose in the k-wave $\left(u_{l}, u_{r}\right)$, the $k$-th elementary waves $\left(u_{k}^{h-1}, u_{k}^{h}\right), h=$ $1,2, \cdots, n_{k}$, are defined as

$$
\begin{gathered}
u_{k}^{0}=u_{l}, \quad u_{k}^{n_{k}}=u_{r}, \\
u_{k}^{h}=\left\{\begin{array}{ll}
R_{k}\left(\alpha_{k}^{h}\right)\left(u_{k}^{h-1}\right), & h \text { is odd, } \\
H_{k}\left(\alpha_{k}^{h}\right)\left(u_{k}^{h-1}\right) & h \text { is even, }
\end{array} \quad\left(h=1,2, \cdots, n_{k}\right) .\right.
\end{gathered}
$$

Notice that the strength of $\alpha_{k}^{h}$ can be zero in the following discussion. Then due to Definition 2.1, these elementary waves satisfy the following monotonicity property:

$$
\begin{array}{cc}
\lambda_{k}\left(u_{k}^{2 p}\right)<\lambda_{k}\left(u_{k}^{2 p+1}\right)=\sigma_{k}\left(u_{k}^{2 p+1}, u_{k}^{2 p+2}\right), & \text { if } \alpha_{k}^{2 p+1} \neq 0, \\
\sigma_{k}\left(u_{k}^{2 p+1}, u_{k}^{2 p+2}\right)=\lambda_{k}\left(u_{k}^{2 p+2}\right)<\lambda_{k}\left(u_{k}^{2 p+3}\right), & \text { if } \alpha_{k}^{2 p+3} \neq 0, \\
2 p+1,2 p+3 \in\left\{1,2, \cdots, n_{k}\right\} . &
\end{array}
$$

We can construct the wave curve $W_{i}\left(s, u_{0}\right)$ as the curve consisting of all the end states that can be connected to $u_{0}$ by admissible shocks, rarefaction waves or their combination of the i-th family. Here $s$ is a non-degenerate parameter along the curve. Up to a linear transformation, this parameter can be chosen as the i-th component of $u$, i.e. $u^{i}$. Then we have the following regularity result.

Lemma 2.1. [2] With the assumption (B), the admissible $i$-th curve $W_{i}\left(s, u_{0}\right)$ has Lipschitz continuous first order derivatives.

To define the approximate solutions, we use the deterministic version of Glimm scheme[13, 21]. As in the classical Glimm scheme, we divide the $(x, t)$ 
plane with grid sizes $\mathrm{r}$ and s satisfying the CFL condition, that is, $\frac{r}{s}>\sup _{i}\left|\lambda_{i}(u)\right|$ for all $u$ under consideration. And pick the pre-chosen random number sequence $\left\{\theta_{m}\right\}_{m=1}^{\infty}$. Then we construct the approximate solution $u_{\theta, r}(x, t)$ inductively:

- At $t=0$, let $u_{\theta, r}(x, 0)=u_{0}(i r), \quad$ for $(i-1) r<x<(i+1) r, \quad i$ odd.

- Suppose $u_{\theta, r}(x, t)$ is defined for $t<j s$, then

$u_{\theta, r}(x, j s)=u_{\theta, r}\left(\left(i+\left(2 \theta_{j}-1\right)\right) r, j s-0\right),(i-1) r<x<(i+1) r, i+j$ odd.

Note that $u_{\theta, r}(x, j s)$ is a piecewise constant function with possible jumps at $x=i r$, where $i+j$ even.

- Now for $i+j$ even, in $j s \leq t<(j+1) s,(i-1) r<x<(i+1) r$, define $u_{\theta, r}(x, t)$ as the solution to the Riemann problem

$$
\begin{cases}u_{t}+f(u)_{x}=0, & (i-1) r<x<(i+1) r, j s \leq t, \\ u(x, j s)=u_{\theta, r}(x, j s) & (i-1) r<x<(i+1) r, i+j \text { even. }\end{cases}
$$

Then the approximate solution can be defined up to $t<(j+1) s$.

The approximate solution can be well defined if the uniform bound of the total variation is obtained. For this purpose, one has to investigate the wave interaction. In $[22,25]$, the following Glimm type functional is defined.

$$
F_{o}(J) \equiv L(J)+M Q_{o}(J)
$$

where the subscript "o" refers to the old one, compared with the new one we shall define later. In the above definition,

$$
\begin{aligned}
L(J)= & \sum\{|\alpha|: \alpha \text { any wave crossing } \mathrm{J}\}, \quad Q_{o}(J)=Q_{d}(J)+Q_{o s}(J), \\
Q_{d}(J)= & \sum\{|\alpha||\beta|: \text { interacting waves } \alpha \text { and } \beta \text { of distinct } \\
& \quad \text { characteristic field crossing } \mathrm{J}\}, \\
Q_{o s}(J)= & \sum_{i=1}^{n} Q_{o s}^{i}, \\
Q_{o s}^{i} & =\sum\{|\alpha||\beta| \max \{-\Theta(\alpha, \beta), 0\}: \alpha \text { and } \beta \text { i-waves crossing J, }
\end{aligned}
$$$$
\alpha \text { to the left of } \beta \text {. }
$$

Here $M$ is a sufficiently large constant, $J$ is any space-like curve. An $i$-wave $\alpha_{i}$ on the left and a $j$-wave $\beta_{j}$ on the right are said to be approaching, if $i>j$. 
And $\Theta(\alpha, \beta)$, called the effective angle between waves $\alpha$ and $\beta$ of the same $\mathrm{i}$-th family, is defined as follows:

$$
\Theta(\alpha, \beta) \equiv \theta_{\alpha}^{+}+\theta_{\beta}^{-}+\sum \theta_{\gamma} .
$$

$\theta_{\alpha}^{+}$represents the value of $\lambda_{i}$ at the right state of $\alpha$ minus its wave speed if $\alpha$ is a shock and is set to be zero if it is a rarefaction wave. Similarly the term $\theta_{\beta}^{-}$ denotes the difference between the speed of $\beta$ and the value of $\lambda_{i}$ at its left end state. $\theta_{\gamma}$ is the value of $\lambda_{i}$ at the right state of the wave $\gamma$ minus that of the left state. The sum $\sum \theta_{\gamma}$ is over the i-waves $\gamma$ between $\alpha$ and $\beta$. When $\Theta(\alpha, \beta)$ is positive, the two waves will not likely to meet; when $\Theta(\alpha, \beta)$ is negative, the two waves may eventually meet and interact.

In the deterministic version of Glimm scheme, all the waves in the solution are partitioned into small subwaves as follows.

Definition 2.2. [25] Let $u_{r} \in W_{i}\left(u_{l}\right)$ so that $u_{l}$ is connected to $u_{r}$ by $i$ discontinuities $\left(u_{j-1}, u_{j}\right)$, and i-rarefaction waves $\left(u_{j}, u_{j+1}\right), j$ odd, $1 \leq j \leq$ $m-1, u_{0}=u_{l}$ and $u_{m}=u_{r}$. A set of vectors $\left\{v_{0}, v_{1}, \cdots, v_{p}\right\}$ is a partition of $\left(u_{l}, u_{r}\right)$ if

(i) $v_{0}=u_{l}, v_{p}=u_{r}, v_{k-1}^{i} \leq v_{k}^{i}, k=1,2, \cdots, p$,

(ii) $\left\{u_{0}, u_{1}, \cdots, u_{m}\right\} \subset\left\{v_{0}, v_{1}, \cdots, v_{p}\right\}$,

(iii) $v_{k} \in R_{i}\left(u_{j}\right), j$ odd, if $u_{j}^{i}<v_{k}^{i}<u_{j+1}^{i}$,

(iv) $v_{k} \in D_{i}\left(u_{j-1}, u_{j}\right), j$ odd, if $u_{j-1}^{i}<v_{k}^{i}<u_{j}^{i}$. Here

$$
\begin{gathered}
D_{i}\left(u_{l}, u_{r}\right) \equiv\left\{u:\left(u-u_{l}\right) \sigma\left(u_{l}, u_{r}\right)-\left(f(u)-f\left(u_{l}\right)\right)=c(u) r_{i}(u)\right. \\
\text { for some scalar } c(u)\} .
\end{gathered}
$$

Then set

(1) $y_{k} \equiv v_{k}-v_{k-1}$,

(2) $\lambda_{i, k} \equiv \lambda_{i}\left(v_{k-1}\right)$ and

$\left[\lambda_{i}\right]_{k} \equiv\left[\lambda_{i}\right]\left(v_{k-1}, v_{k}\right) \equiv \lambda_{i}\left(v_{k}\right)-\lambda_{i}\left(v_{k-1}\right)>0$ if (iii) holds,

(3) $\lambda_{i, k} \equiv \sigma\left(u_{j-1}, u_{j}\right)$ and $\left[\lambda_{i}\right]_{k} \equiv\left[\lambda_{i}\right]\left(v_{k-1}, v_{k}\right) \equiv 0$ if (iv) holds. 
In the following, we always assume that a rarefaction wave is divided into several small rarefaction shocks with strength less than the grid size of Glimm scheme. Then the shock waves and rarefaction waves can be treated similarly after the wave partition. Due to the regularity of composite wave curve, i.e. Lemma 2.1, such partition is stable under the perturbation in the following sense.

Lemma 2.2. [25] Suppose that $u_{r} \in W_{i}\left(u_{l}\right), \bar{u}_{r} \in W_{i}\left(\bar{u}_{l}\right)$, with $u_{r}^{i}-u_{l}^{i}=$ $\bar{u}_{r}^{i}-\bar{u}_{l}^{i} \equiv \alpha>0$, and $\left|u_{l}-\bar{u}_{l}\right| \equiv \beta$. Then there exist partitions $\left\{v_{0}, v_{1}, \cdots, v_{p}\right\}$ and $\left\{\bar{v}_{0}, \bar{v}_{1}, \cdots, \bar{v}_{p}\right\}$ for the $i$-waves $\left(u_{l}, u_{r}\right)$ and $\left(\bar{u}_{l}, \bar{u}_{r}\right)$ respectively such that $\bar{v}_{k}^{i}-\bar{v}_{0}^{i}=v_{k}^{i}-v_{0}^{i}, k=1,2, \cdots, p$, and the following holds:

(i) $\sum_{k=1}^{p}\left|y_{k}-\bar{y}_{k}\right|=0(1) \alpha \beta$,

(ii) $\left|\lambda_{i, k}-\bar{\lambda}_{i, k}\right|=0(1) \beta, k=1,2, \cdots, p$,

(iii) Let $\Theta^{+}\left(u_{l}, u_{r}\right)$ represents the value of $\lambda_{i}$ at the right state $u_{r}$ minus the wave speed of the right-most $i$-wave in $\left(u_{l}, u_{r}\right)$. Similar definition holds for $\Theta^{-}\left(u_{l}, u_{r}\right)$. Then

$$
\left|\Theta^{-}\left(u_{l}, u_{r}\right)-\Theta^{-}\left(\bar{u}_{l}, \bar{u}_{r}\right)\right|+\left|\Theta^{+}\left(u_{l}, u_{r}\right)-\Theta^{+}\left(\bar{u}_{l}, \bar{u}_{r}\right)\right|=0(1) \alpha \beta .
$$

Moreover, the index set $\{1,2, \cdots, p\}$ can be written as a disjoint union of subsets I, II and III such that

(iv) for $k \in I$ corresponding to rarefaction waves, both $v_{k}$ and $\bar{v}_{k}$ are of type (iii) of Definition 2.2 and

$$
\sum_{k \in I}\left|\left[\lambda_{i}\right]_{k}-\left[\bar{\lambda}_{i}\right]_{k}\right|=0(1) \alpha \beta
$$

(v) for $k \in I I$ corresponding to discontinuities, both $v_{k}$ and $\bar{v}_{k}$ are of the type (iv) of Definition 2.2.

(vi) for $k \in I I I$ corresponding to mixed types, $v_{k}$ and $\bar{v}_{k}$ are of different types and

$$
\sum_{k \in I I I}\left|\left[\lambda_{i}\right]_{k}+\left[\bar{\lambda}_{i}\right]_{k}\right|=0(1) \alpha \beta .
$$

Here $\Theta^{+}\left(u_{l}, u_{r}\right)$ represents the value of $\lambda_{i}$ at the right state $u_{r}$ minus the wave speed of the rightest $i$-wave in $\left(u_{l}, u_{r}\right)$. Similar definition holds for $\Theta^{-}\left(u_{l}, u_{r}\right)$. 
This lemma describes the $C^{2}$ like dependency of the Riemann problem on the end states. Then the effect of wave interaction can be estimated by the Glimm functional and the cancellation as in [25].

Lemma 2.3. [25] Let $u_{l}, u_{m}$ and $u_{r}$ be three nearby states and $\left(u_{i-1}, u_{i}\right)\left(v_{i-1}, v_{i}\right)$, $i=1,2, \cdots, n$, be $i$-waves in the Riemann problem $\left(u_{l}, u_{m}\right)$ and $\left(u_{m}, u_{r}\right)$ respectively with the partition defined in Definition 2.2. Here, rarefaction waves are divided into small rarefaction shocks with strength less than the grid size s in $t$ direction. Then the wave partition of the $i$-wave $\left(w_{i-1}, w_{i}\right), i=1,2, \cdots, n$, in the Riemann problem $\left(u_{l}, u_{r}\right)$ is the linear superposition of the above two solutions modulo the nonlinear effect of the order $s, Q\left(u_{l}, u_{m}, u_{r}\right)$ and $\delta C\left(u_{l}, u_{m}, u_{r}\right)$, where $\delta=\left|u_{m}-u_{l}\right|+\left|u_{r}-u_{m}\right|$. In other words,

$$
\begin{gathered}
\gamma_{i}=\alpha_{i}+\beta_{i}+O(1)\left(\delta C\left(u_{l}, u_{m}, u_{r}\right)+Q_{o}\left(u_{l}, u_{m}, u_{r}\right)+s\right), \\
\eta\left(\gamma_{i}\right)=\eta\left(\alpha_{i}\right)+\eta\left(\beta_{i}\right)+O(1)\left(\delta C\left(u_{l}, u_{m}, u_{r}\right)+Q_{o}\left(u_{l}, u_{m}, u_{r}\right)+s\right),
\end{gathered}
$$

with

$$
\begin{gathered}
\alpha_{i}=\sum_{k=1}^{n_{\alpha_{i}}} \alpha_{i, k}=u_{i}^{i}-u_{i-1}^{i} \beta_{i}=\sum_{k=1}^{n_{\beta_{i}}} \beta_{i, k}=v_{i}^{i}-v_{i-1}^{i} \text {, and } \gamma_{i}=w_{i}^{i}-w_{i-1}^{i}, \\
\eta\left(\alpha_{i}\right)=\sum_{k=1}^{n_{\alpha_{i}}} \eta\left(\alpha_{i, k}\right), \text { with } \eta\left(\alpha_{i, k}\right)=\alpha_{i, k} \lambda_{i, k}, \\
\text { similar definition for } \eta\left(\beta_{i}\right) \text { and } \eta\left(\gamma_{i}\right), \\
C\left(u_{l}, u_{m}, u_{r}\right) \equiv \sum_{i=1}^{n} C^{i}\left(u_{l}, u_{m}, u_{r}\right)=\frac{1}{2}|| \gamma_{i}|-| \alpha_{i}|-| \beta_{i}||,
\end{gathered}
$$

for some constants $n_{\alpha_{i}}$ and $n_{\beta_{i}}, i=1,2 \cdots, n$. Each $\alpha_{i, k}=\left(u_{i, k-1}, u_{i, k}\right)$ and $\beta_{i, k}=\left(v_{i, k-1}, v_{i, k}\right)$ is a shock or a rarefaction shock. $C\left(u_{l}, u_{m}, u_{r}\right)$ measures the amount of cancellation.

The above wave interaction estimate is crucial in the study of conservation laws under general assumption (B). Compared with the parallel estimate under condition (A), the error is bounded by at least of cubic order terms instead of quadratic ones. This seems better when the total variation of the approximate solutions is small. On the other hand, it implies that the decrease of $Q_{o}$ after the wave interaction in the same family is much less than the decrease in the classical Glimm functional under the condition (A). This fact causes difficulty in the proof of consistency and wave tracing argument. For example, one has to 
divide waves into two groups by checking whether the total strength of the involving waves is greater than a pre-chosen small constant or not. We include the following estimate from [25] by using the old Glimm functional for comparison with Theorem 2.2 given later.

Lemma 2.4. [25] Let $\epsilon$ be a constant with $\frac{1}{2}<\epsilon<1$. The waves in an approximate solution in a given a time zone $\Lambda=\{(x, t):-\infty<x<\infty, M s \leq$ $t<(M+N) s\}$, can be partitioned into subwaves of categories I, II or III with the following properties:

(i). The subwaves in I are surviving. Given a subwave $\alpha(t), M s \leq t<$ $(M+N) s$, in $I$, write $\alpha \equiv \alpha(M s)$ and denote by $|\alpha(t)|$ its strength at time $t$, by $[\sigma(\alpha)]$ the variation of its speed and by $[\alpha]$ the variation of the jump of the states across it over the time interval $M s \leq t<(M+N) s$. Then

$$
\sum_{\alpha \in I}([\alpha]+|\alpha(M s)|[\sigma(\alpha)])=O(1)\left(D_{o}(\Lambda)(N s)^{-\epsilon}+T \cdot V \cdot N^{1+\epsilon} s^{\epsilon}+s\right) .
$$

(ii). A subwave $\alpha(t)$ in II has positive initial strength $|\alpha(M s)|>0$, but is cancelled in the zone $\Lambda,|\alpha((M+N) s)|=0$. Moreover, the total strength and variation of the wave speed satisfy

$$
\begin{gathered}
\sum_{\alpha \in I I}([\alpha]+|\alpha(t)|)=O(1)\left(D_{o}(\Lambda)+s\right), M s \leq t<(M+N) s, \\
\sum_{\alpha \in I I}([\alpha]+|\alpha(M s)|[\sigma(\alpha)]) \leq 0(1)\left(D_{o}(\Lambda)(N s)^{-\epsilon}+T \cdot V \cdot N^{1+\epsilon} s^{\epsilon}+s\right) .
\end{gathered}
$$

(iii). A subwave in III has zero initial strength $|\alpha(M s)|=0$, and is created in the zone $\Lambda,|\alpha((M+N) s)| \neq 0$. Moreover, the total strength and variation of the wave speed satisfy

$$
\begin{gathered}
\sum_{\alpha \in I I I}([\alpha]+|\alpha(t)|)=O(1)\left(D_{o}(\Lambda)+s\right), M s \leq t<(M+N) s, \\
\sum_{\alpha \in I I I}([\alpha]+|\alpha((M+N) s)|[\sigma(\alpha)]) \leq 0(1)\left(D_{o}(\Lambda)(N s)^{-\epsilon}+T \cdot V \cdot N^{1+\epsilon} s^{\epsilon}+s\right) .
\end{gathered}
$$

Here $D_{o}(\Lambda)=F_{o}(M s)-F_{o}((M+N) s)$, and T.V. $=$ Tot.Var. $\left\{u_{0}(x)\right\}$. And $F(t)$ is the Glimm functional on the space-like curve at time $t$.

Moreover, the functional defined in (2.4) can not be reduced to the one defined in [13] even if each characteristic field is genuinely nonlinear or linearly degenerate because they are not of the same order. 
To overcome these difficulties, we define a new Glimm functional as follows.

$$
F(J) \equiv L(J)+M Q_{n}(J),
$$

where

$$
\begin{gathered}
Q_{n}(J)=Q_{d}(J)+Q_{n s}(J), \quad Q_{n s}(J)=\sum_{i=1}^{n} Q_{n s}^{i}, \\
Q_{n s}^{i}=\sum\left\{|\alpha||\beta| \frac{\max \{-\Theta(\alpha, \beta), 0\}}{t . v \cdot(\alpha, \beta)_{i}}: \alpha \text { and } \beta \text { i-waves crossing } \mathrm{J},\right. \\
\alpha \text { to the left of } \beta\},
\end{gathered}
$$

where t.v. $(\alpha, \beta)_{i}=\sum\{|\gamma|: \gamma$ any i-wave crossing $\mathrm{J}$ and between $\alpha$ and $\beta$ including $\alpha$ and $\beta$ \}.

$Q_{d}, L(J)$ and $M$ are defined as before.

For this new functional, we shall first prove that $F$ is non-increasing so that the uniform bound on the total variation of the approximate solution follows.

Theorem 2.1. $J_{1}$ and $J_{2}$ are two spaced-like curves and $J_{2}$ is the immediate successor of $J_{1}$. Suppose that $F\left(J_{1}\right)$ is sufficiently small, then the following estimate holds.

$$
F\left(J_{2}\right)-F\left(J_{1}\right) \leq 0
$$

The proof of this theorem will be given in the next section.

By the Lipschitz dependency of the wave speed on the left and right states, it is easy to see that the new functional is equivalent to the classical Glimm functional in the sense of the decreasing order when the characteristic field is genuinely nonlinear. Thus, it gives a better way to control the error of wave tracing argument than the old Glimm functional in the general setting. Furthermore, we have the following clear estimate of wave tracing argument.

Theorem 2.2. The waves in an approximate solution in a given a time zone $\Lambda=\{(x, t):-\infty<x<\infty, M s \leq t<(M+N) s\}$, can be partitioned into subwaves of categories I, II or III with the following properties:

(i). The subwaves in I are surviving. Given a subwave $\alpha(t), M s \leq t<$ $(M+N) s$, in $I$, write $\alpha \equiv \alpha(M s)$ and denote by $|\alpha(t)|$ its strength at time $t$, 
by $[\sigma(\alpha)]$ the variation of its speed and by $[\alpha]$ the variation of the jump of the states across it over the time interval $M s \leq t<(M+N) s$. Then

$$
\sum_{\alpha \in I}([\alpha]+|\alpha(M s)|[\sigma(\alpha)])=O(1)(D(\Lambda)+s) .
$$

(ii). A subwave $\alpha(t)$ in II has positive initial strength $|\alpha(M s)|>0$, but is cancelled in the zone $\Lambda,|\alpha((M+N) s)|=0$. Moreover, the total strength and variation of the wave speed satisfy

$$
\begin{gathered}
\sum_{\alpha \in I I}([\alpha]+|\alpha(t)|)=O(1)(D(\Lambda)+s), M s \leq t<(M+N) s, \\
\sum_{\alpha \in I I}([\alpha]+|\alpha(M s)|[\sigma(\alpha)]) \leq 0(1)(D(\Lambda)+s) .
\end{gathered}
$$

(iii). A subwave in III has zero initial strength $|\alpha(M s)|=0$, and is created in the zone $\Lambda,|\alpha((M+N) s)| \neq 0$. Moreover, the total strength and variation of the wave speed satisfy

$$
\begin{gathered}
\sum_{\alpha \in I I I}([\alpha]+|\alpha(t)|)=O(1)(D(\Lambda)+s), M s \leq t<(M+N) s, \\
\sum_{\alpha \in I I I}([\alpha]+|\alpha((M+N) s)|[\sigma(\alpha)]) \leq 0(1)(D(\Lambda)+s) .
\end{gathered}
$$

Here $D(\Lambda)=F(M s)-F((M+N) s)$, and T.V. $=$ Tot.Var. $\left\{u_{0}(x)\right\}$. And $F(t)$ is the Glimm functional on the space-like curve at time $t$.

The proof of Theorem 2.2 will be given in the last section.

\section{$3 \quad$ Wave Interaction Estimates}

In this section we show that the new Glimm functional is decreasing due to the wave interaction. To do so, we check two typical cases first. All the other cases can be dealt with similar argument.

Proof of Theorem 2.1. Case (I)(cf. Fig.1): Suppose that there are three waves $\alpha, \beta, \varepsilon$ are i-shocks or rarefaction shocks. $\beta$ interacts with $\varepsilon$ at time $t$ without any cancellation:

$$
\beta+\varepsilon \rightarrow \gamma+\sum_{k \neq i} \delta_{k}
$$




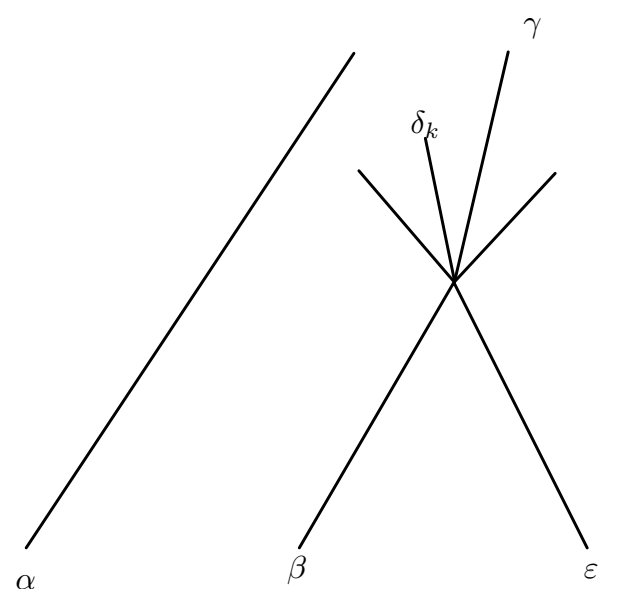

Figure 1: Case I

From the definition of effective angle, $\Theta(\beta, \varepsilon) \leq 0$. Since there is no cancellation, for simplicity, we assume $\alpha, \beta, \varepsilon \geq 0$, that is, they are in the same direction.

By the monotonicity property (2.3), we also assume, without loss of generality that the generated i-wave $\gamma$ is a single shock and

$$
\Theta(\alpha, \varepsilon) \leq 0
$$

From the standard wave interaction estimate, we know the difference of the functional $L$ crossing the time $t$ is

$$
\Delta L \equiv L(t+)-L(t-)=O(1) \beta \varepsilon(-\Theta(\beta, \varepsilon)) .
$$

We only need to estimate the change of $Q_{n s}$ because the estimation on other parts are the same as those for the classical Glimm functional.

Before the wave interaction at time $t$, the potential wave interaction $Q_{n s}$ is

$$
Q_{n s}(t-)=\frac{\alpha \varepsilon(-\Theta(\alpha, \varepsilon))}{\text { t.v. }(\alpha, \varepsilon)_{i}}+\frac{\beta \varepsilon(-\Theta(\beta, \varepsilon))}{\text { t.v. }(\beta, \varepsilon)_{i}}+\frac{\alpha \beta(\max \{-\Theta(\alpha, \beta), 0\})}{\text { t.v. }(\alpha, \beta)_{i}} .
$$

After the wave interaction, it becomes

$$
Q_{n s}(t+)=\frac{\alpha \gamma(\max \{-\Theta(\alpha, \gamma), 0\})}{t . v \cdot(\alpha, \gamma)_{i}}
$$


Then

$$
\begin{aligned}
& \Delta Q_{n s} \equiv Q_{n s}(t+)-Q_{n s}(t-) \\
= & -\frac{\beta \varepsilon(-\Theta(\beta, \varepsilon))}{t \cdot v \cdot(\beta, \varepsilon)_{i}}+\left[\frac{\alpha \gamma(\max \{-\Theta(\alpha, \gamma), 0\})}{t . v \cdot(\alpha, \gamma)_{i}}-\frac{\alpha \varepsilon(-\Theta(\alpha, \varepsilon))}{t . v \cdot(\alpha, \varepsilon)_{i}}-\frac{\alpha \beta(\max \{-\Theta(\alpha, \beta), 0\})}{t . v \cdot(\alpha, \beta)_{i}}\right] \\
= & I+I I .
\end{aligned}
$$

We may assume $\Theta(\alpha, \gamma) \leq 0$. Otherwise it is easy to see that

$$
\Delta Q_{n s} \leq-\frac{\beta \varepsilon(-\Theta(\beta, \varepsilon))}{t . v \cdot(\beta, \varepsilon)_{i}} .
$$

By Lemma 2.3, we have

$$
\begin{gathered}
\gamma=\beta+\varepsilon+O(1) \beta \varepsilon(-\Theta(\beta, \varepsilon)), \\
\sigma(\gamma) \gamma=\sigma(\beta) \beta+\sigma(\varepsilon) \varepsilon+O(1) \beta \varepsilon(-\Theta(\beta, \varepsilon)) .
\end{gathered}
$$

Thus,

$$
\begin{gathered}
t . v \cdot(\alpha, \varepsilon)_{i}=t . v \cdot(\alpha, \beta)_{i}+\varepsilon, \\
t . v \cdot(\alpha, \gamma)_{i}=t . v \cdot(\alpha, \beta)_{i}+\varepsilon+O(1) \beta \varepsilon(-\Theta(\beta, \varepsilon)), \\
-\Theta(\alpha, \gamma)=-\Theta(\alpha, \beta)+\sigma(\beta)-\sigma(\gamma), \\
-\Theta(\alpha, \varepsilon)=-\Theta(\alpha, \beta)+\sigma(\beta)-\sigma(\varepsilon) .
\end{gathered}
$$

It implies that

$$
\begin{aligned}
I I \leq & \frac{\alpha \gamma(-\Theta(\alpha, \gamma))}{t \cdot v \cdot(\alpha, \varepsilon)_{i}}\left[1+O(1) \frac{\beta \varepsilon(-\Theta(\beta, \varepsilon))}{t \cdot v \cdot(\alpha, \varepsilon)_{i}}\right]-\frac{\alpha \varepsilon(-\Theta(\alpha, \varepsilon))}{t \cdot v \cdot(\alpha, \varepsilon)_{i}}-\frac{\alpha \beta(\max \{-\Theta(\alpha, \beta), 0\})}{t \cdot v \cdot(\alpha, \beta)_{i}} \\
= & \frac{\alpha[\gamma(-\Theta(\alpha, \gamma))-\varepsilon(-\Theta(\alpha, \varepsilon))]}{t \cdot v \cdot(\alpha, \varepsilon)_{i}}+O(1) \frac{\alpha[\beta \varepsilon(-\Theta(\beta, \varepsilon))]}{t \cdot v \cdot(\alpha, \varepsilon)_{i}}-\frac{\alpha \beta(\max \{-\Theta(\alpha, \beta), 0\})}{t \cdot v \cdot(\alpha, \beta)_{i}} \\
= & \frac{\alpha[\gamma(-\Theta(\alpha, \beta)+\sigma(\beta)-\sigma(\gamma))-\varepsilon(-\Theta(\alpha, \beta)+\sigma(\beta)-\sigma(\varepsilon))]}{t \cdot v \cdot(\alpha, \varepsilon)_{i}} \\
& -\frac{\alpha \beta(\max \{-\Theta(\alpha, \beta), 0\})}{t \cdot v \cdot(\alpha, \beta)_{i}}+O(1) \frac{\alpha[\beta \varepsilon(-\Theta(\beta, \varepsilon))]}{t \cdot v \cdot(\alpha, \varepsilon)_{i}} \\
= & \frac{\alpha[\beta(-\Theta(\alpha, \beta))+O(1) \beta \varepsilon(-\Theta(\beta, \varepsilon))]}{t \cdot v \cdot(\alpha, \varepsilon)_{i}} \\
& -\frac{\alpha \beta(\max \{-\Theta(\alpha, \beta), 0\})}{t \cdot v \cdot(\alpha, \beta)_{i}}+O(1) \frac{\alpha[\beta \varepsilon(-\Theta(\beta, \varepsilon))]}{t \cdot v \cdot(\alpha, \varepsilon)_{i}} \\
\leq & O(1) \frac{\alpha[\beta \varepsilon(-\Theta(\beta, \varepsilon))]}{t \cdot v \cdot(\beta, \varepsilon)_{i}} .
\end{aligned}
$$

In the last inequality, we have used the fact that t.v. $(\alpha, \varepsilon)_{i} \geq t \cdot v \cdot(\alpha, \beta)_{i}$ and t.v. $(\alpha, \varepsilon)_{i} \geq t . v \cdot(\beta, \varepsilon)_{i}$. 
Therefore, when the total variation of the approximate solution is sufficiently small, we deduce that

$$
\begin{aligned}
\Delta Q_{n s} & \leq-\frac{[\beta \varepsilon(-\Theta(\beta, \varepsilon))]}{t \cdot v \cdot(\beta, \varepsilon)_{i}}+O(1) \frac{\alpha[\beta \varepsilon(-\Theta(\beta, \varepsilon))]}{t \cdot v \cdot(\beta, \varepsilon)_{i}} \\
& \leq-\frac{1}{2} \frac{[\beta \varepsilon(-\Theta(\beta, \varepsilon))]}{t . v \cdot(\beta, \varepsilon)_{i}} .
\end{aligned}
$$

Combined with (3.1), this implies that $F$ is decreasing for suitably chosen constant $M$

$$
\Delta F \leq 0 .
$$

Case (II)(cf. Fig.2): Assume that $\alpha, \beta$ are i-waves and $\gamma$ is $\mathrm{j}$-waves $(i>j)$. Without loss of generality, we assume that they are shocks or rarefaction shocks. Furthermore, we assume that $\alpha, \beta$ do not interact with each other before the time $t$, but after the interaction at time $t$

$$
\beta+\gamma \longrightarrow \bar{\beta}+\bar{\gamma}+\sum_{k \neq i, j} \delta_{k},
$$

$\bar{\beta}$ interacts with $\alpha$. Again we may assume that no cancellation happens and $\alpha, \beta, \gamma \geq 0$.
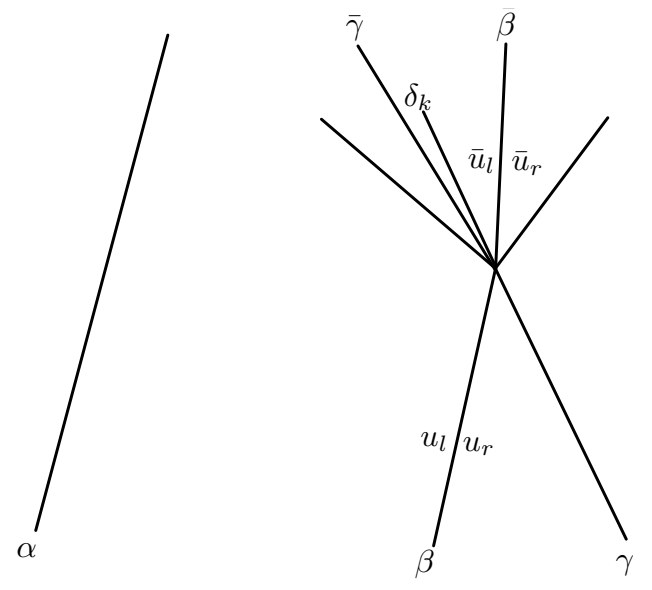

Figure 2: Case II

In this case, the standard wave interaction estimates yield

$$
\Delta L \equiv L(t+)-L(t-)=O(1)|\beta||\gamma| .
$$


Again we need to estimate the change of $Q_{n}$.

Before the wave interaction at time $t$, the potential wave interaction $Q_{n}$ is

$$
Q_{n}(t-)=\beta \gamma+\alpha \gamma
$$

After the wave interaction, the $i$-wave $\bar{\beta}$ may be a composite wave. We partition it into $\bar{\beta}_{1}, \cdots, \bar{\beta}_{m}$ as in Lemma 2.3 with intermediate states $\bar{u}_{l}=\bar{u}_{0}, \bar{u}_{1}, \cdots, \bar{u}_{m}=$ $\bar{u}_{r}$. The wave interaction potential $Q_{n}$ now becomes

$$
Q_{n}(t+)=\alpha \bar{\gamma}+\sum_{k=1}^{m} \frac{\bar{\beta}_{k} \alpha\left(\max \left\{-\Theta\left(\alpha, \bar{\beta}_{k}\right), 0\right\}\right)}{t \cdot v \cdot\left(\alpha, \bar{\beta}_{k}\right)_{i}}+\sum_{k<i, k \neq j} \alpha \delta_{k} .
$$

Then the change of $Q_{n}$ is

$$
\Delta Q_{n}=Q_{n}(t+)-Q_{n}(t-)=-\beta \gamma+O(1) \alpha \beta \gamma+\sum_{k=1}^{m} \frac{\bar{\beta}_{k} \alpha\left(\max \left\{-\Theta\left(\alpha, \bar{\beta}_{k}\right), 0\right\}\right)}{t . v \cdot\left(\alpha, \bar{\beta}_{k}\right)_{i}} .
$$

It seems that from the Lipschitz continuity of wave speed on the end states, the third term on the right hand side can only be bounded by $\gamma$. But in fact, with the regularity of composite wave curves, i.e. Lemma 2.1, we can have better estimate. By Lemma 2.2, we partition $\beta$ into small sub-waves $\beta_{1}, \cdots, \beta_{m}$ corresponding to $\bar{\beta}_{1}, \cdots, \bar{\beta}_{m}$. Then we can write $\Theta\left(\alpha, \bar{\beta}_{k}\right)$ in terms of $\Theta\left(\alpha, \beta_{k}\right)$ :

$$
\begin{aligned}
-\Theta\left(\alpha, \bar{\beta}_{k}\right)= & -\Theta\left(\alpha, \beta_{k}\right)+\Theta^{-}\left(u_{l}, u_{r}\right)+\sum_{l=1}^{k-1}\left[\lambda_{i}\right]_{l}+\left[\lambda_{i, k}-\lambda_{i}\left(u_{k-1}\right)\right] \\
& -\Theta^{-}\left(\bar{u}_{l}, \bar{u}_{r}\right)-\sum_{l=1}^{k-1}\left[\bar{\lambda}_{i}\right]_{l}-\left[\bar{\lambda}_{i, k}-\lambda_{i}\left(\bar{u}_{k-1}\right)\right] .
\end{aligned}
$$

As $\alpha, \beta$ do not interact with each other, by definition, we have

$$
-\Theta(\alpha, \beta) \leq 0 .
$$

Moreover, since we assume that $\beta$ is an $\mathrm{i}$-th simple wave, from the Definition 2.2 , it implies that $-\Theta\left(\alpha, \beta_{k}\right)=-\Theta(\alpha, \beta) \leq 0$. Then by applying the Lemma 2.2 and observing that $\left|u_{l}-\bar{u}_{l}\right|=O(1) \gamma$, the effective angle can be estimated as follows.

$$
\max \left\{-\Theta\left(\alpha, \bar{\beta}_{k}\right), 0\right\} \leq O(1) \beta \gamma
$$

Therefore,

$$
\Delta Q_{n} \leq-\beta \gamma+O(1) \alpha \beta \gamma \leq-\frac{1}{2} \beta \gamma
$$


Similar to case (I), this implies that $F$ is decreasing for suitably chosen constant $M$, that is

$$
\Delta F \leq 0
$$

When cancellation happens, we can prove the non-increasing of $F$ in a straightforward way because the amount of cancellation is of first order. So for the general case, we can regard the problem as the superposition of case (I) and (II). By using (3.2) and (3.3) repeatedly, we can show that $F$ is nonincreasing after the wave interaction under the condition that the total variation of the approximate solution is sufficiently small. Thus Theorem 2.1 follows.

\section{Consistency and Convergence Rate}

With the above wave interaction estimates, we can prove Theorem 2.2 on the wave tracing argument.

Proof of Theorem 2.2. It is obvious that $|\alpha(M s)|[\sigma(\alpha)]$ at time $\mathrm{t}$ can be controlled by $O(1)\left(Q_{n}(\Lambda)+C(\Lambda)\right)$ if the wave interaction is between waves of different families or cancellation occurs. We only need to consider the interaction of waves of the same family and with the same sign.

Consider the interaction of two Riemann problems:

$$
\left(u_{l}, u_{m}\right)+\left(u_{m}, u_{r}\right) \longrightarrow\left(u_{l}, u_{r}\right) .
$$

We can assume that $\left(u_{l}, u_{m}\right)$ and $\left(u_{m}, u_{r}\right)$ are connected by several $k$ simple waves $\alpha=\left(\alpha_{k}^{1}, \ldots, \alpha_{k}^{n_{1}}\right)$ and $\beta=\left(\beta_{k}^{1}, \ldots, \beta_{k}^{n_{2}}\right)$ respectively. Furthermore, from the monotonicity property $(2.3)$, we may assume that after interaction $\left(u_{l}, u_{r}\right)$ is resolved by a single k-shock $\gamma$ without loss of generality.

We have the following estimates from the Lemma 2.3 .

$$
\begin{gathered}
\gamma=\sum_{i=1}^{n_{1}} \alpha_{k}^{i}+\sum_{i=1}^{n_{2}} \beta_{k}^{i}+O(1) Q_{o s}(\alpha, \beta), \\
\sigma(\gamma) \gamma=\sum_{i=1}^{n_{1}} \sigma\left(\alpha_{k}^{i}\right) \alpha_{k}^{i}+\sum_{i=1}^{n_{2}} \sigma\left(\beta_{k}^{i}\right) \beta_{k}^{i}+O(1) Q_{o s}(\alpha, \beta), \\
\sigma\left(\alpha_{k}^{1}\right) \leq \sigma\left(\alpha_{k}^{2}\right) \leq \ldots \leq \sigma\left(\alpha_{k}^{n_{1}}\right) \leq \sigma(\gamma) \leq \sigma\left(\beta_{k}^{1}\right) \leq \sigma\left(\beta_{k}^{2}\right) \leq \ldots \leq \sigma\left(\beta_{k}^{n_{2}}\right) .
\end{gathered}
$$


Then we can estimate the variation of the speeds $\left[\sigma\left(\alpha_{k}^{i}\right)\right]$ and $\left[\sigma\left(\beta_{k}^{i}\right)\right]$ as follows.

$$
\begin{aligned}
& \sum_{i=1}^{n_{1}} \alpha_{k}^{i}\left[\sigma\left(\alpha_{k}^{i}\right)\right]+\sum_{i=1}^{n_{2}} \beta_{k}^{i}\left[\sigma\left(\beta_{k}^{i}\right)\right] \\
= & \sum_{i=1}^{n_{1}} \alpha_{k}^{i}\left(\sigma\left(\alpha_{k}^{i}\right)-\sigma(\gamma)\right)+\sum_{i=1}^{n_{2}} \beta_{k}^{i}\left(\sigma\left(\beta_{k}^{i}\right)-\sigma(\gamma)\right) \\
\equiv & I+I I .
\end{aligned}
$$

And

$$
\begin{aligned}
I= & \sum_{i=1}^{n_{1}} \alpha_{k}^{i}\left\{\sigma\left(\alpha_{k}^{i}\right)-\frac{1}{\gamma}\left[\sum_{i=1}^{n_{1}} \sigma\left(\alpha_{k}^{i}\right) \alpha_{k}^{i}+\sum_{i=1}^{n_{2}} \sigma\left(\beta_{k}^{i}\right) \beta_{k}^{i}+O(1) Q_{o s}(\alpha, \beta)\right]\right\} \\
= & \sum_{i=1}^{n_{1}} \frac{\alpha_{k}^{i}}{t \cdot v \cdot(\alpha, \beta)_{i}}\left[\sum_{j=1}^{n_{1}} \alpha_{k}^{j}\left(\sigma\left(\alpha_{k}^{i}\right)-\sigma\left(\alpha_{k}^{j}\right)\right)+\sum_{l=1}^{n_{2}} \beta_{k}^{l}\left(\sigma\left(\alpha_{k}^{i}\right)-\sigma\left(\beta_{k}^{l}\right)\right)+O(1) Q_{o s}(\alpha, \beta)\right] \\
= & \frac{\left[\sum_{i, j=1}^{n_{1}} \alpha_{k}^{i} \alpha_{k}^{j}\left(\sigma\left(\alpha_{k}^{i}\right)-\sigma\left(\alpha_{k}^{j}\right)\right)+\sum_{i=1}^{n_{1}} \sum_{l=1}^{n_{2}} \alpha_{k}^{i} \beta_{k}^{l}\left(\sigma\left(\alpha_{k}^{i}\right)-\sigma\left(\beta_{k}^{l}\right)\right)\right]}{t \cdot v \cdot(\alpha, \beta)_{i}} \\
& +\frac{\sum_{i=1}^{n_{1}} \alpha_{k}^{i} O(1) Q_{o s}(\alpha, \beta)}{t \cdot v \cdot(\alpha, \beta)_{i}} \\
= & \frac{\left[\sum_{i=1}^{n_{1}} \sum_{l=1}^{n_{2}} \alpha_{k}^{i} \beta_{k}^{l}\left(\sigma\left(\alpha_{k}^{i}\right)-\sigma\left(\beta_{k}^{l}\right)\right)\right]}{t \cdot v \cdot(\alpha, \beta)_{i}}+\frac{\sum_{i=1}^{n_{1}} \alpha_{k}^{i} O(1) Q_{o s}(\alpha, \beta)}{t \cdot v \cdot(\alpha, \beta)_{i}}
\end{aligned}
$$

since the summation $\sum_{i, j=1}^{n_{1}} \alpha_{k}^{i} \alpha_{k}^{j}\left(\sigma\left(\alpha_{k}^{i}\right)-\sigma\left(\alpha_{k}^{j}\right)\right)=0$.

Similarly

$$
I I=\frac{\left[\sum_{i=1}^{n_{1}} \sum_{l=1}^{n_{2}} \alpha_{k}^{i} \beta_{k}^{l}\left(\sigma\left(\alpha_{k}^{i}\right)-\sigma\left(\beta_{k}^{l}\right)\right)\right]}{t . v \cdot(\alpha, \beta)_{i}}+\frac{\sum_{i=1}^{n_{1}} \alpha_{k}^{i} O(1) Q_{o s}(\alpha, \beta)}{t \cdot v \cdot(\alpha, \beta)_{i}}
$$

Therefore, we have

$$
I+I I=\left(2+\sum_{i=1}^{n_{1}} \alpha_{k}^{i}\right) \frac{Q_{o s}(\alpha, \beta)}{t \cdot v \cdot(\alpha, \beta)_{i}}=O(1) Q_{n s}(\alpha, \beta)
$$

Then Theorem 2.2 follows.

With Theorem 2.2, we can prove the consitency of Glimm scheme clearly, i.e. we want to show that the following term

$$
\int_{-\infty}^{\infty} \int_{0}^{\infty}\left(u \phi_{t}+f(u) \phi_{x}\right)(x, t) d x d t+\int_{-\infty}^{\infty}(u \phi)(x, 0) d x
$$




$$
=\sum_{k=0}^{M N} \int_{-\infty}^{\infty}(u(x, k s+0)-u(x, k s-0)) \phi(x, k s) d x,
$$

vanishes as the grid size $r$ tends to zero. Here $\phi(x, t)$ is the test function with compact support, $\phi(x, t)=0, t>T=M N s$.

As in [24] we may start with the simple example of one shock. Denote by $x=x(k) r$ the location of the shock at time $t=k s$. We have

$$
\begin{aligned}
& \int_{-\infty}^{\infty}(u(x, k s+0)-u(x, k s-0)) \phi(x, k s) d x \\
& = \begin{cases}\int_{x(k) r}^{x(k) r+\sigma s}\left(u_{+}-u_{-}\right) \phi(x, k s) d x, & \text { if } \theta_{k} r>\sigma s, \\
\int_{x(k) r+\sigma s}^{(x(k)+1) r}\left(u_{-}-u_{+}\right) \phi(x, k s) d x, & \text { if } \theta_{k} r<\sigma s .\end{cases}
\end{aligned}
$$

If the test function is a constant $\phi_{0}$ then the error becomes, for the interval $I=(0, \sigma s / r)$,

$$
\begin{aligned}
& \sum_{k=0}^{M N} \int_{-\infty}^{\infty}(u(x, k s+0)-u(x, k s-0)) \phi(x, k s) d x \\
& =\phi_{0}\left(u_{+}-u_{-}\right)\left(B(M N, I)(r-\sigma s)-B\left(M N, I^{c}\right) \sigma s\right) \\
& =\phi_{0}\left(u_{+}-u_{-}\right) T\left(B(M N, I)\left(\frac{r}{s}-\sigma\right)-(M N-B(M N, I)) \sigma\right) \frac{1}{M N} \\
& =\phi_{0}\left(u_{+}-u_{-}\right) T\left(\frac{B(M N, I)}{M N}-\sigma \frac{s}{r}\right) \frac{r}{s},
\end{aligned}
$$

which tends to zero as $M N \rightarrow \infty$ when the random sequence is equidistributed as (1.6).

The non-constancy of the test function $\phi(x, t)$ gives the error of the order $O(1) L N s=O(1) L T / M$, if we divide the time zone $0 \leq t<T=M N s$ into $M$ small time zones $N(l-1) s \leq t<N l s, l=1,2, \cdots, M$. Here $L$ is the Lipschitz constant of $\phi(x, t)$. Then it tends to zero as $M \rightarrow \infty$.

For a general solution, the speed of the shocks or rarefaction shocks is changing. The variation of the speed has been discussed in Theorem 2.2, which says that for a surviving wave $\alpha$, its strength $|\alpha|$ times the variation of its speed in a time zone $\Lambda_{l}$ is of the order of $D\left(\Lambda_{l}\right)+s$. Thus the error contributed by surviving subwaves in a given time zone $\Lambda_{l}$ is $O(1)\left(D\left(\Lambda_{l}\right)+s\right) N s$. The total error of this kind over $0 \leq t<T$ is then $E_{1}=O(1)\left(D(t \geq 0) \frac{T}{M}+s T\right)$. Similarly, the error contributed by the cancelled subwaves in $0 \leq t \leq T$ is

$$
E_{2}=O(1)(D(t \geq 0)+s T) .
$$

Thus the total error is of the form

$$
E=O(1)\left[(B(N, I) / N-|I|) T+s T+D(t \geq 0) \frac{T}{M}\right],
$$


which tends to zero as $M, N \rightarrow \infty$. This means that the approximate solutions constructed by the deterministic version of Glimm scheme converges to a weak solution to the Cauchy problem as the grid size tends to zero.

Finally, we prove Theorem 1.1 on the convergence rate of Glimm scheme. In fact, after the preparation in above sections, the proof can follow the argument in [6] for systems satisfying condition (A). For completeness, we outline the proof as follows by using the new Glimm functional.

Proof of Theorem 1.1. Consider the approximate solution up to time $T=\bar{m} s+$ $s^{\prime}$, where $s^{\prime} \in[0, \epsilon), s$ is the grid size in $x$ and $t$. Pick a constant $\delta \gg s$. Divide $[0, T]$ into finitely many intervals $J_{i} \equiv\left[m_{i} s, m_{i+1} s\right], i=0,1, \cdots$. Let $m_{0}=0$. Construct $J_{i}$ and a subset $E$ of $\mathbb{N}$ inductively as follows.

1. If $F\left(m_{i} s\right)-F\left(\left(m_{i}+1\right) s\right) \leq \delta$, then let $m_{i+1}$ be the largest integer such that $F\left(m_{i} s\right)-F\left(\left(m_{i+1} s\right)\right) \leq \delta$ and $m_{i+1} s-m_{i} s \leq \delta$. And denote $i \in E$.

2. If $F\left(m_{i} s\right)-F\left(\left(m_{i}+1\right) s\right)>\delta$, then let $m_{i+1}=m_{i}+1$. And denote $i \in E^{c}$.

Then there exists some finite number $\mu \leq \bar{m}$ such that $m_{\mu}=\bar{m}$. Since $T$ is fixed and $F(t)$ is uniformly bounded,

$$
\# E \leq O(1) \frac{1}{\delta}, \# E^{c} \leq O(1) \frac{1}{\delta} .
$$

For each interval $J_{i}, i \in E, i \leq \mu$, define an auxiliary piecewise constant function $w(x, t)$ with the following property:

- Every subwave $\alpha(t)$ in $u$ corresponds a wave front in $w$ with the same initial and final position.

- The wave front in $w$ has constant wave strength and speed.

Applying the wave tracing result Theorem 2.2,

$$
\begin{aligned}
& \left\|w\left(\cdot, m_{i+1} s\right)-S_{m_{i+1} s-m_{i} s} w\left(\cdot, m_{i} s\right)\right\|_{L_{1}} \\
= & O(1)\left(m_{i+1} s-m_{i} s\right)\left\{\left(F\left(m_{i} s\right)-F\left(m_{i+1} s\right)\right)\right\} \\
& +O(1)\left(m_{i+1} s-m_{i} s\right)\left\{s+\frac{1+\ln \left(m_{i+1}-m_{i}\right)}{m_{i+1}-m_{i}}\right\} .
\end{aligned}
$$

From the structure of $w$, we also obtain

$$
\left\|u\left(\cdot, m_{i+1} s\right)-w\left(\cdot, m_{i+1} s\right)\right\|_{L_{1}}=0(1)\left(F\left(m_{i} s\right)-F\left(m_{i+1} s\right)\right)\left(m_{i+1} s-m_{i} s\right),
$$


Thus, by combining (4.4) and (4.5), for each $i \in E$, we have

$$
\begin{aligned}
& \left\|u\left(\cdot, m_{i+1} s\right)-S_{m_{i+1} s-m_{i} s} u\left(\cdot, m_{i} s\right)\right\|_{L_{1}} \\
\leq & O(1)\left(m_{i+1} s-m_{i} s\right)\left\{\left(F\left(m_{i} s\right)-F\left(m_{i+1} s\right)\right)\right. \\
& \left.+\left[s+\frac{1+\ln \left(m_{i+1}-m_{i}\right)}{m_{i+1}-m_{i}}\right]\right\} .
\end{aligned}
$$

And for each $i \in E^{c}$, by the Lipschitz continuity of approximate solution constructed by the Glimm scheme, we have

$$
\left\|u\left(\cdot, m_{i+1} s\right)-S_{m_{i+1} s-m_{i} s} u\left(\cdot, m_{i} s\right)\right\|_{L_{1}} \leq O(1) s
$$

By applying these two estimates, (4.3), the construction of $J_{i}$ and the property of standard Riemann semigroup, the $L_{1}$ distance between $u(x, T)$ and $S_{T} u(x, 0)$ can be controlled by

$$
\begin{aligned}
& \left\|u(\cdot, T)-S_{T} u(\cdot, 0)\right\|_{L_{1}} \\
\leq & \sum_{i=0}^{\mu-1}\left\|S_{T-m_{i+1} s} u\left(\cdot, m_{i+1} s\right)-S_{T-m_{i} s} u\left(\cdot, m_{i} s\right)\right\|_{L_{1}} \\
& +\left\|u(\cdot, T)-S_{T-\bar{m} s} u(\cdot, \bar{m} s)\right\|_{L_{1}} \\
\leq & L \sum_{i=0}^{\mu-1}\left\|u\left(\cdot, m_{i+1} s\right)-S_{m_{i+1} s-m_{i} s} u\left(\cdot, m_{i} s\right)\right\|_{L_{1}}+O(1) s^{\prime} \\
\leq & O(1) \sum_{i \in E}\left\{( m _ { i + 1 } s - m _ { i } s ) \left\{\left(F\left(m_{i} s\right)-F\left(m_{i+1} s\right)\right)\right.\right. \\
& \left.+\left[s+\frac{1+\ln \left(m_{i+1}-m_{i}\right)}{m_{i+1}-m_{i}}\right]\right\}+O(1) \sum_{i \in E^{c}} s \\
\leq & O(1)\left\{\delta+s+\frac{s}{\delta}\left(2+\left|\ln \frac{\delta}{s}\right|\right)\right\} .
\end{aligned}
$$

Let $\delta=s^{k} \ln (|\ln s|), k \in(0,1)$. Then simple computation yields that when $k=\frac{1}{2}$, the convergence rate is $o(1) s^{\frac{1}{2}}|\ln s|$. This completes the proof.

Acknowledgment: The research was supported by the RGC Competitive Earmarked Research Grant of Hong Kong, CityU \#103607. 


\section{References}

[1] Bianchini, S. and Bressan, A., Vanishing viscosity solutions of nonlinear hyperbolic systems. Ann. of Math. (2) 161 (2005), no. 1, 223-342.

[2] Bianchini, S., On the Riemann Problem for Non-Conservative Hyperbolic Systems, Arch. Rational Mech. Anal, 166(2003), 1-26

[3] Bressan, A. and Colombo, R.M., The semigroup generated by $2 \times 2$ conservation laws, Arch. Rational Mech. Anal., 133, 1-75, 1995.

[4] Bressan, A., Hyperbolic systems of conservation laws. The one-dimensional Cauchy problem. Oxford Lecture Series in Mathematics and its Applications, 20(2000).

[5] Bressan, A., Crasta, Graziano and Piccoli, Benedetto, Well-posedness of the Cauchy problem for $n \times n$ systems of conservation laws. Mem. Amer. Math. Soc. 146 (2000), no. 694

[6] Bressan, A., Marson A., Error bounds for a deterministic version of the Glimm scheme, Arch. Rational Mech. Anal. 142 (1998), no. 2, 155-176.

[7] Bressan, A., Liu, T.-P., and Yang, T., $L_{1}$ stability estimates for $n \times n$ conservation laws, Arch. Ration. Mech. Anal. 149, no. 1, 1-22, 1999.

[8] Bressan, A., The unique limit of the Glimm scheme, Arch.Rational Mech.Anal. 130(1995), 205-230.

[9] Alberto Bressan and Tong Yang, On the convergence rate of vanishing viscosity approximations, Communications on Pure and Applied Mathematics, LVII(2004), 1075-1109.

[10] Chern, I.-L., Local and global interaction for nongenuinely nonlinear hyperbolic conservation laws, Indiana Univ. Math. J. 49 (2000), no. 3, 1199-1227.

[11] Courant, R. and Friedrichs, K.O., "Supersonic flow and shock waves", Springer-Verlag, 1948.

[12] R. J. DiPerna, Convergence of approximate solutions to conservation laws, Arch. Rational Mech. Anal. 82 (1983), 27-70. 
[13] Glimm, J., Solutions in the large for nonlinear hyperbolic systems of equations, Comm. Pure Appl. Math. 18(1965), 697-715.

[14] Glimm, J., and Lax, P.D, Decay of solutions of systems of hyperbolic conservation laws, Memoirs Amer. Math. Soc., 101, 1970.

[15] Jiale Hua and Tong Yang, An improved convergence rate of Glimm scheme for general systems of hyperbolic conservation laws, Journal of Differential Equations, 231(2006), 92-107.

[16] Hua, L.-K. and Wang Y., Applications of number theory to numerical analysis, Springer-Verlag, 1981.

[17] Kruzkov, S., First-order quasilinear equations with several space variables, Mat. Sb. 123, 228-255, 1970; English transl. in Math. USSR Sb. 10, 217$273,1970$.

[18] N. N. Kuznetsov, Accuracy of some approximate methods for computing the weak solutions of a first-order quasi-linear equation, U.S.S.R. Comp. Math. and Math. Phys. 16 (1976), 105-119.

[19] Lax, P.D., Hyperbolic systems of conservation laws II, Comm. Pure Appl. Math. 10(1957), 53 7-566.

[20] LeFloch. P. and Iguchi, T., Existence theory for hyperbolic systems of conservation laws with general flux-functions, Arch. Ration. Mech. Anal. 168 (2003), no. 3, 165-244.

[21] Liu, T.-P., The deterministic version of the Glimm scheme, Comm. Math. Phys., 57(1977), $135-148$.

[22] Liu, T.-P., Admissible solutions of hyperbolic conservation laws, Memoirs of Amer. Meth. Soc., Vol 30, 240(1981).

[23] Liu, T.-P., The entropy condition and the admissibility of shocks, J. Math. Anal. Appl., 53(1976), 78-88.

[24] Liu, T.-P. and Yang, T., Well-posedness theory for hyperbolic conservation laws, Comm. Pure Appl. Math., 52, no. 12, 1553-1586, 1999.

[25] Liu, T.-P. and Yang, T., Weak solutions of general systems of hyperbolic conservation laws, Commun. Math. Phys. 230, 289-327(2002). 
[26] Oleinik, O., Uniqueness and stability of the generalized solution of the Cauchy problem for a quasilinear equation, Usp. Mat. Nauk 14, 165-170, 1959; Amer. Math. Soc. Transl. Ser. 2, 33, 285-290, 1964.

[27] Smoller, J., "Shock waves and reaction-diffusion equations, SpringerVerlag", New York, 1983.

[28] T. Tang and Z. H. Teng, The sharpness of Kuznetsov's $O(\sqrt{\Delta x}) L^{1}$ error estimate for monotone difference schemes, Math. Comp. 64 (1995), 581-589.

[29] Yang, T., Convergence rate of Glimm scheme for general systems of hyperbolic conservation laws, Taiwanese journal of mathematics, Vol 7(2003), No 2, 195-205 